\title{
Grape seed procyanidin B2 ameliorates hepatic lipid metabolism disorders in $\mathrm{db} / \mathrm{db}$ mice
}

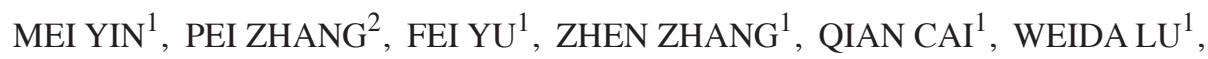

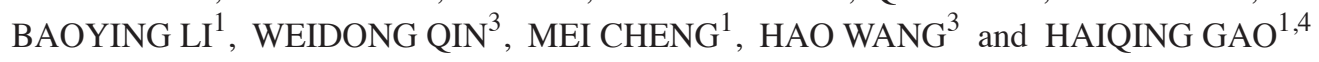

${ }^{1}$ Department of Geriatrics, Qilu Hospital of Shandong University, Jinan, Shandong 250012; ${ }^{2}$ Department of Endocrinology, Liaocheng People's Hospital Affiliated to Taishan Medical College, Liaocheng, Shandong 252000; ${ }^{3}$ Department of Critical Care Medicine, Qilu Hospital of Shandong University; ${ }^{4}$ Key Laboratory of Cardiovascular Proteomics of Shandong Province Department of Geriatrics, Qilu Hospital of Shandong University, Jinan, Shandong 250012, P.R. China

Received June 16,2016; Accepted April 27, 2017

DOI: $10.3892 / \mathrm{mmr} .2017 .6900$

\begin{abstract}
Diabetes is commonly associated with liver lipid metabolism disorders. AMP-activated protein kinase (AMPK) has a key role in regulating lipid metabolism. Grape seed procyanidin B2 (GSPB2), a natural polyphenol polymer, ameliorates mitochondrial dysfunction and inhibits oxidative stress or apoptosis via AMPK pathways. In the present study, the hypothesis that GSPB2 treatment may ameliorate liver lipid metabolic disorders by activating AMPK and downstream pathways was tested in diabetic mice. $\mathrm{Db} / \mathrm{m}$ mice were used as controls, and diabetic $\mathrm{db} / \mathrm{db}$ mice were randomly divided into 2 groups for treatment: Vehicle and GSPB2 (30 mg/kg/day for 10 weeks). Animals were weighed every week. Fasting blood was collected prior to sacrifice to measure fasting blood glucose (FBG), triglycerides (TG) and total cholesterol (TC). Hepatic TG and free fatty acid (FFA) levels were analyzed. Hepatic sections were examined by light microscopy following hematoxylin and eosin staining. The expression of hepatic AMPK, phosphorylated acetyl-CoA carboxylase (ACC), carnitine palmitoyl transferase 1 (CPT1) and 4-hydroxynonenal (4-HNE) was measured by western blot analysis. Liver mitochondria were isolated to assess electron transport complex I (CI), complex II (CII) and complex IV by high-resolution respirometry. The results demonstrated that GSPB2 significantly decreased body weight and serum TG, TC and FFA levels, but not FBG levels in diabetic mice.
\end{abstract}

Correspondence to: Dr Hao Wang, Department of Critical Care Medicine, Qilu Hospital of Shandong University, 107 Wenhua-xi Road, Jinan, Shandong 250012, P.R. China

E-mail:wanghao34@126.com

Dr Haiqing Gao, Department of Geriatrics, Qilu Hospital of Shandong University, 107 Wenhua-xi Road, Jinan, Shandong 250012, P.R. China

E-mail: 13573187788@163.com

Key words: grape seed procyanidin B2, diabetes, liver, lipid metabolism, AMP-activated protein kinase
GSPB2 visibly decreased lipid droplet accumulation in the liver and significantly reduced hepatic TG and FFA levels. In diabetic mice, GSPB2 restored liver AMPK and ACC phosphorylation, increased CPT1 protein expression, ameliorated lipid peroxidation damage, which was assessed by comparing 4-HNE levels, and partially restored the damaged mitochondrial respiratory capacity of CI and CII in the liver. In conclusion, long-term oral treatment with GSPB2 may benefit hepatic lipid metabolism disorders, potentially by decreasing hepatic lipid synthesis and increasing hepatic FFA $\beta$-oxidation via the AMPK-ACC pathway.

\section{Introduction}

Diabetes has become a global health epidemic. In 2012, the economic burden of diagnosed diabetes was estimated to be in excess of \$245 billion in the United States alone, representing a $>40 \%$ increase in cost over 2007 estimates, with most of this burden attributed to the treatment and management of diabetes $(1,2)$. Diabetes is commonly associated with lipid metabolism disorders and abnormal serum lipid levels, which accelerate the progression of diabetes and lead to atherosclerosis and cardiovascular diseases $(3,4)$, the primary causes of death in patients with diabetes. The liver has a pivotal role in regulating lipid metabolism, accounting for the production and degradation of fatty acid (FA), cholesterol, glycolipids, ketone bodies, phospholipids, steroids and triacylglycerols (5). Fatty liver disease is highly prevalent in patients with type 2 diabetes mellitus (6). Increased circulating levels of free FAs (FFAs) lead to increased delivery of FFAs to the liver, which subsequently drive the synthesis of excess triglycerides (TG) in the liver; the accumulation of excess liver fat is worsened by impaired hepatic FA oxidation in patients with type 2 diabetes (5). Ameliorating hepatic lipid metabolism disorders may be an effective way of improving whole-body lipid metabolism, decelerating the progression of diabetes complications and improving the prognosis of patients with diabetes.

AMP-activated protein kinase (AMPK), a heterotrimeric serine-threonine kinase, is an important cellular energy sensor in the majority of tissues (7). A previous study demonstrated that dysfunction of hepatic AMPK in diabetes represents a key 
mechanism for hepatic lipid accumulation and hyperlipidemia associated with diabetes (8). AMPK phosphorylation in the liver results in the stimulation of FA oxidation and inhibition of lipogenesis $(9,10)$; active AMPK phosphorylates and inactivates certain rate-limiting enzymes in the liver that are associated with lipolysis, such as acetyl-CoA carboxylase (ACC). ACC catalyzes malonyl CoA synthesis, which is a major building block for de novo FA synthesis and also functions as an allosteric inhibitor of carnitine palmitoyl transferase 1 (CPT1) (10). Unphosphorylated ACC inhibits CPT1, which is the rate-limiting enzyme responsible for the transfer of long-chain fatty acyl CoA to the mitochondria for $\beta$-oxidation (11). Therefore, AMPK activation is an important therapeutic target in hepatic lipid metabolism disorders and hyperlipidemia, specifically in diabetes (12).

Procyanidins are a complex family of polyphenol polymers that are present in a wide variety of natural products, including grape wines, fruits and vegetables. Grape seed proanthocyanidin extracts (GSPEs) have been demonstrated to exhibit a variety of potent pharmacological activities, including functions against oxidative stress, inflammation and atherosclerosis $(13,14)$. Grape seed procyanidin B2 (GSPB2) is one of the major components of GSPEs and possesses similar pharmacological activities. Furthermore, in diabetes models, GSPEs have lipid-lowering (15) and hepatocyte protective effects (16), and lead to the activation of AMPK; GSPE treatment has been demonstrated to ameliorate mitochondrial dysfunction and inhibit oxidative stress or apoptosis in mesangial cells treated with high-dose glucosamine (17) or in diabetic nephropathy (18), via AMPK-dependent signaling. Considering that inactivation of hepatic AMPK is a key event in the pathogenesis of hyperlipidemia in diabetes (12), GSPB2 may be a useful agent to ameliorate liver lipid metabolism disorders and to improve hyperlipidemia in diabetes. To the best of our knowledge, this hypothesis has not been previously investigated.

The present study aimed to evaluate the effect of GSPB2 on liver lipid metabolism in the $\mathrm{db} / \mathrm{db}$ diabetic mouse model and the potential underlying mechanism. The $\mathrm{db} / \mathrm{db}$ mice are a well-established animal model for the investigation of diabetic complications. We hypothesize that GSPB2 may ameliorate liver lipid metabolic disorders in $\mathrm{db} / \mathrm{db}$ mice via the activation of AMPK and downstream pathways.

\section{Materials and methods}

Animals and treatments. Male C57BLKS/J db/db and db/m mice ( $\mathrm{n}=24 ; 7$ weeks old; average weight, $32.1 \mathrm{~g}$ ) were purchased from the Model Animal Research Center of Nanjing University (Nanjing, China). They were housed in standard animal cages and received laboratory pellet chow and tap water ad libitum in a constant environment (room temperature, $20-22^{\circ} \mathrm{C}$; humidity, 40-60\%) with a 12-h light/dark cycle. All experimental procedures were approved by the Animal Ethics Committee of Shandong University (Jinan, China). Mice were adapted for one week prior to initiation of the study. Age-matched $\mathrm{db} / \mathrm{m}$ mice were used as a control group (Control, n=8). GSPB2 (>90\% pure) was purchased from Tianjin Jianfeng Natural Produce R\&D Co., Ltd (Tianjin, China). $\mathrm{Db} / \mathrm{db}$ mice were randomly divided into two groups for treatment ( $\mathrm{n}=8$ each): Vehicle (DM group; normal saline solution) and GSPB2 (DMT group; $30 \mathrm{mg} / \mathrm{kg}$ body weight per day in normal saline solution orally for 10 weeks). Each group was observed between weeks 8 and 18 of age without any other intervention. Animals were weighed each week. At the end of the intervention, all mice were fasted overnight and sacrificed. Fasting blood was collected and the liver tissue was dissected. The sera and tissues were stored at $-80^{\circ} \mathrm{C}$.

Measurement of body weight, fasting blood glucose (FBG) and serum lipids. Animals were weighed every week. Fasting blood was collected prior to sacrifice and centrifuged at 7,700 x g for $10 \mathrm{~min}$ at $4^{\circ} \mathrm{C}$ to measure $\mathrm{FBG}, \mathrm{TG}$ and total cholesterol (TC) using an automatic biochemistry and analysis instrument (ADVIA-1650 autoanalyzer; Bayer AG, Leverkusen, Germany). Serum FFA levels were determined with a FFA Detection kit using the acylCoA synthetase-acylCoA oxidase (ACS-ACOD) method, according to the manufacturer's protocol (Wako Pure Chemical Industries, Ltd., Osaka, Japan).

Hepatic lipid analysis. The stored liver samples (100 mg) were lysed and homogenized in $2 \mathrm{ml}$ of a solution containing $150 \mathrm{mmol} / \mathrm{l} \mathrm{NaCl}, 0.1 \%$ Triton X-100 and $10 \mathrm{mmol} / 1$ Tris, using a polytron homogenizer (cat. no. NS-310E; Microtec Co., Ltd., Chiba, Japan) for $1 \mathrm{~min}$ at room temperature. Liver TG level was analyzed with a Tissue triglyceride assay kit using the glycerol phosphate oxidase-peroxidase method (Applygen Technologies, Beijing, China) and normalized to protein levels measured using a bicinchoninic acid (BCA) Protein Assay kit (Beyotime Institute of Biotechnology, Haimen, China). Liver homogenate FFA level was determined with a FFA Detection kit using the ACS-ACOD method (Wako Pure Chemical Industries, Ltd.) and normalized to protein levels, as previously described (19).

Hepatic pathological examination. The excised parts of livers were immediately fixed in $4 \%$ paraformaldehyde at room temperature for $12 \mathrm{~h}$ and embedded in paraffin. After solidification, 5- $\mu \mathrm{m}$ sections were cut from blocks. After hematoxylin and eosin (H\&E) staining, sections were examined by light microscopy at a magnification of x100.

Western blot analysis. Western blot analysis was performed on samples of livers obtained from the three groups of mice. Mice livers were homogenized in radioimmunoprecipitation lysis buffer (Beyotime Institute of Biotechnology), sonicated for $20 \mathrm{sec}$ at $4^{\circ} \mathrm{C}$ and normalized with the BCA Protein Assay kit (Beyotime Institute of Biotechnology). Equal amounts of protein $(50 \mathrm{mg}$ ) were separated by $10 \%$ SDS-PAGE, transferred to polyvinylidene difluoride membranes, incubated with blocking buffer (5\% non-fat dry milk and $0.05 \%$ Tween-20 in TBS) for $1 \mathrm{~h}$ at room temperature and probed with antibodies for phosphorylated AMPK (1:1,000 dilution; cat. no. \#5256; Cell Signaling Technology, Inc., Danvers, MA, USA), AMPK (1:1,000 dilution; cat. no. \#2532; Cell Signaling Technology, Inc.), phosphorylated ACC (1:1,000 dilution; cat. no. \#3661; Cell Signaling Technology, Inc.), CPT1 (1:1,000 dilution; cat. no. ab128568; Abcam, Cambridge, UK), 4-hydroxynonenal (4-HNE, 1:1,000 dilution; cat.no.ab46545; Abcam) andGAPDH (1:1,000 dilution; cat. no. \#2118; Cell Signaling Technology, Inc.) overnight at $4^{\circ} \mathrm{C}$. Membranes were subsequently incubated with horseradish peroxidase-conjugated goat 
A

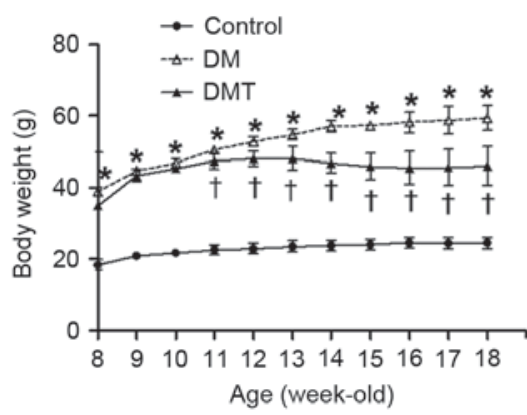

C

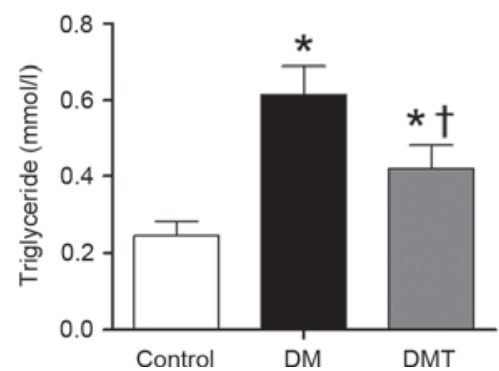

B
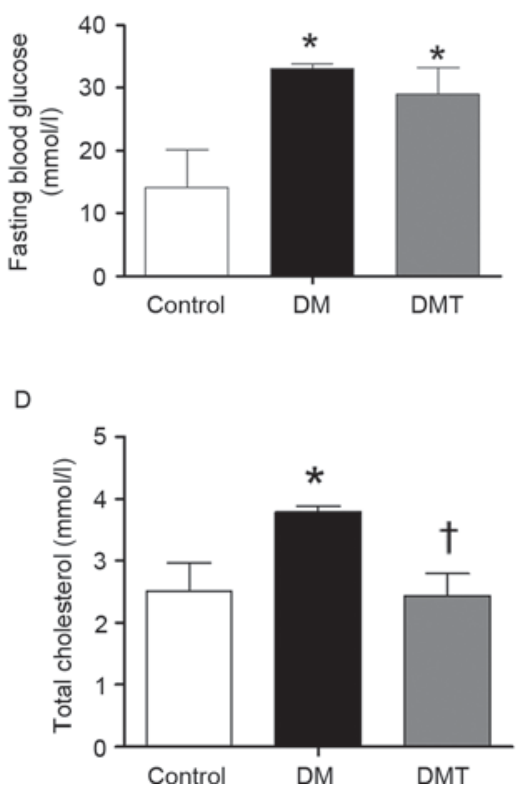

E

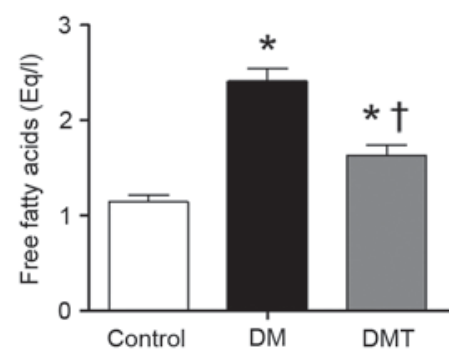

Figure 1. GSPB2 decreases the body weight and serum lipid levels of diabetic mice. (A) Body weight was measured in control, DM and DMT mice each week and for the 10-week duration of the GSPB2 oral administration study (30 mg/kg/day). (B) Fasting blood glucose, (C) serum triglycerides, (D) total cholesterol and (E) free fatty acid levels in the serum of control, DM and DMT groups. Data are presented as the mean \pm standard error ( $\mathrm{n}=8$ per group). ${ }^{*}<0.05$ vs. control and ${ }^{\dagger} \mathrm{P}<0.05$ vs. DM. GSPB2, grape seed procyanidin B2; DM, diabetic mice treated with vehicle alone; DMT, DM treated with GSPB2.

anti-rabbit or anti-mouse (1:4,000 dilution; cat. nos. ZB-2301 and ZB-2305; ZSGB-BIO; OriGene Technologies, Inc., Beijing, China) as secondary antibodies for $2 \mathrm{~h}$ at room temperature and visualized by chemiluminescence immunoblotting detection (Amersham Imager 600; GE Healthcare Bio-Sciences, Pittsburgh, PA, USA). The intensity of each protein band was quantified by densitometry using ImageJ software (version 1.48; National Institutes of Health, Bethesda, MD, USA).

Isolation of liver mitochondria. Liver tissue (0.5-0.8 g; n=4 per group) was excised immediately after mice were sacrificed and immersed in ice-cold mitochondrial isolation buffer [MIB; $210 \mathrm{mM}$ mannitol, $70 \mathrm{mM}$ sucrose, $10 \mathrm{mM}$ HEPES, $1 \mathrm{mM}$ EDTA; final pH, 7.2; with $0.5 \%$ FA-free bovine serum albumin (BSA)]. Tissue was minced and homogenized with additional MIB and $0.5 \%$ BSA by using a Potter Elvehjem homogenizer and loose-fitting Teflon pestle. Mitochondrial isolation involved differential centrifugation at $4^{\circ} \mathrm{C}$, as previously described (20). The mitochondrial pellet was resuspended in MIB without BSA and centrifuged for an additional $10 \mathrm{~min}$ at $9,600 \mathrm{x}$ g at $4^{\circ} \mathrm{C}$ for further mitochondrial purification. The final mitochondrial pellet was resuspended in MIB and the protein concentration was determined by the Biuret method (20).

Mitochondrial respiratory capacity. Oxygen consumption was measured by high-resolution respirometry (Oroboros Instruments, Innsbruck, Austria). A standard substrate/inhibitor titration protocol was used as described previously (21) for functional analysis of mitochondrial respiratory-chain complexes after adding isolated mitochondria $(0.15 \mathrm{mg})$ to respiration medium [110 $\mathrm{mM}$ mannitol, $0.5 \mathrm{mM}$ EGTA, $3 \mathrm{mM} \mathrm{MgCl2}, 20 \mathrm{mM}$ taurine $10 \mathrm{mM} \mathrm{KH} 2 \mathrm{PO} 4,60 \mathrm{mM} \mathrm{K}$ lactobionate, $0.3 \mathrm{mM}$ DTT and $0.1 \%$ BSA (FA-free), adjusted to $\mathrm{pH} 7.1 ; 37^{\circ} \mathrm{C}$ ] (22). Briefly, after stabilization (3-5 min), real-time oxygen concentration and flux data were collected sequentially. Complex I (CI)-dependent mitochondrial respiration was induced by adding glutamate $(10 \mathrm{mM})$, malate (5 mM) and ADP (1 mM). Complex II (CII)-dependent respiration was induced by adding rotenone $(0.5 \mu \mathrm{M})$ to selectively inhibit CI, followed by succinate $(10 \mathrm{mM})$, which is a CII substrate. Antimycin A $(5 \mu \mathrm{M})$ was then added to inhibit CIII, followed by the addition of TMPD $(0.5 \mathrm{mM})$ and ascorbate $(2 \mathrm{mM})$ as artificial electron donors for CIV. To ensure that the respiratory capacity of CIV was not limited by cytochrome c depletion, respiration was measured after the addition of cytochrome c $(10 \mu \mathrm{M})$.

Statistical analysis. Statistical analysis was performed using SPSS 16.0 (SPSS Inc., Chicago, IL, USA) and GraphPad Prism 4 (Graphpad Software Inc., La Jolla, CA, USA). Data were analyzed by one-way analysis of variance followed by a least significant difference post hoc test. Results are presented as the mean \pm standard error of the mean, unless otherwise stated. $\mathrm{P}<0.05$ was considered to indicate a statistically significant difference.

\section{Results}

GSPB2 decreases the body weight and serum lipid levels in diabetic mice. DM and DMT mice had substantially more body weight compared with age-matched control mice at the beginning of the study ( 8 weeks of age; $\mathrm{P}<0.05$; Fig. 1A). Four weeks later however (starting at 11 weeks of age and throughout the study duration), GSPB2 treatment significantly decreased the body weight of DMT group compared with the DM group $(\mathrm{P}<0.05$; Fig. 1A). FBG was significantly higher in $\mathrm{DM}$ and 
A

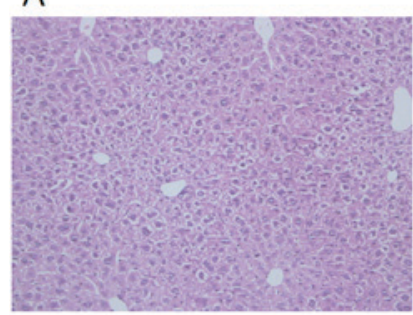

D
B

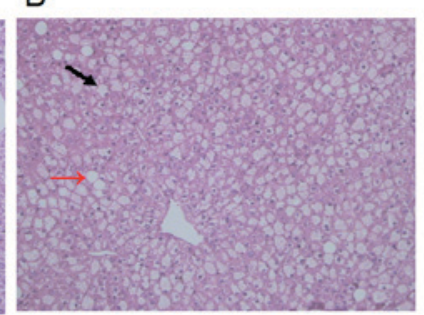

E
C

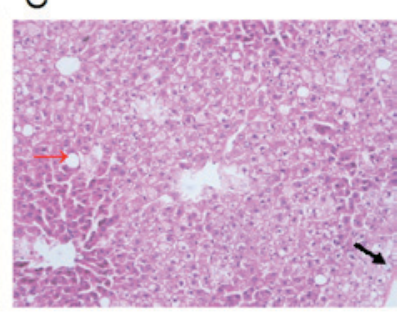

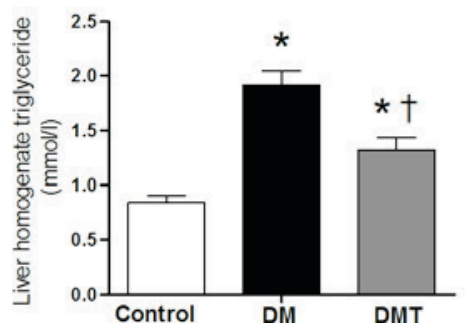

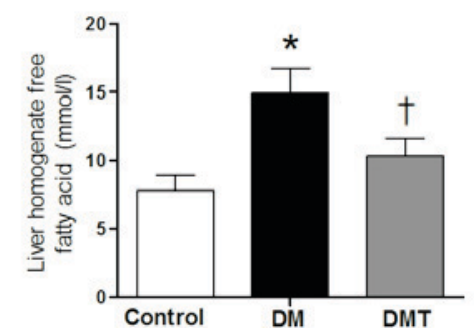

Figure 2. GSPB2 decreases lipid droplet accumulation and hepatic lipid levels. Histological examination of liver tissue (magnification, x100) after hematoxylin and eosin staining in (A) control, (B) DM and (C) DMT mice. The red arrows indicate lipid droplets and the black arrows indicate ballooning degeneration in hepatic cells. The levels of (D) triglycerides and (E) free fatty acid in the liver homogenates of control, DM and DMT mice were measured by glycerol phosphate oxidase-peroxidase and acylCoA synthetase-acylCoA oxidase methods. Data are presented as the mean + standard error ( $\mathrm{n}=8$ per group). ${ }^{*} \mathrm{P}<0.05$ vs. control and ${ }^{\dagger} \mathrm{P}<0.05$ vs. DM. GSPB2, grape seed procyanidin B2; DM, diabetic mice treated with vehicle alone; DMT, DM treated with GSPB2.

DMT mice compared with control mice $(\mathrm{P}<0.05$; Fig. 1B), and GSPB2 treatment did not significantly affect FBG levels compared with the DM group ( $\mathrm{P}>0.05$; Fig. 1B). Compared with control mice, the DM group exhibited higher serum levels of TG ( $\mathrm{P}<0.05$; Fig. 1C), TC $(\mathrm{P}<0.05$; Fig. 1D) and FFA $(\mathrm{P}<0.05$; Fig. 1E). GSPB2 treatment significantly decreased the TG, TC and FFA levels compared with the DM group (all $\mathrm{P}<0.05$ ), however, serum TG and FFA levels remained higher compared with control mice in the DMT group (Fig. 1C-E).

GSPB2 decreases lipid droplet accumulation and hepatic lipid levels. H\&E-stained paraffin sections revealed normal hepatic architecture with clear hepatic lobule, radial liver cell cord and clear hepatic sinusoid in livers of control mice (Fig. 2A). By contrast, livers of the DM group exhibited pathological symptoms: Accumulation of lipid droplets in the cytoplasm and ballooning degeneration (Fig. 2B). After mice were fed GSPB2 for 10 weeks, this hepatocellular damage was ameliorated (Fig. 2C). Changes in the liver homogenate lipid levels appeared to be similar to serum lipid levels. The DM group exhibited significantly higher liver homogenate TG and FFA levels compared with controls (both $\mathrm{P}<0.05$; Fig. 2D and E), which were significantly decreased by GSPB2 treatment (both $\mathrm{P}<0.05$; Fig. 2D and E).

GSPB2 restores liver AMPK and ACC phosphorylation levels, increases CPT1 levels and ameliorates lipid peroxidation damage. The phosphorylation levels of AMPK and ACC were lower in the DM group compared with the control group (both $\mathrm{P}<0.05$; Fig. 3). Following GSPB2 treatment, the phosphorylation of AMPK and ACC was significantly increased compared with the DM group (both $\mathrm{P}<0.05$; Fig. 3). Furthermore, CPT1 protein levels were also significantly higher in the DMT group compared with the DM group ( $\mathrm{P}<0.05$; Fig. 4A). 4-HNE protein levels, which are markers of cellular lipid peroxidation damage, were significantly increased in the DM group compared with control $(\mathrm{P}<0.05)$, but were significantly reduced following GSPB2 treatment $(\mathrm{P}<0.05$; Fig. 4B).

GSPB2 restores damaged liver mitochondrial respiratory capacity. Liver samples from DM mice exhibited a significant decrease in the respiration function of all mitochondrial complexes (CI, CII and CIV), compared with controls (all $\mathrm{P}<0.05$; Fig. 5). However, GSPB2 treatment significantly restored the respiratory capacities of CI and CII in the DMT group compared with the DM group (both $\mathrm{P}<0.05$; Fig. 5). The respiratory capacity of CIV was also increased by GSPB2 treatment in the DMT group compared with the DM group, however, this increase was not significant ( $\mathrm{P}>0.05$; Fig. 5).

\section{Discussion}

GSPB2 is a dimeric form of GSPE, an extract compound from grape seeds with established lipid-lowering, anti-atherosclerosis and hepatocyte protective properties $(15,16,23,24)$. Previous studies from our group have demonstrated the protective effects of GSPB2 on diabetic complications, including diabetic nephropathy and diabetic arterial damage $(23,24)$. As diabetes is frequently complicated by hepatic lipid metabolism disorders and high lipid levels, in the present study, we hypothesized that GSPB2 treatment may alleviate hepatic lipid metabolism disorders and lower serum lipid levels in diabetes. Long-term oral treatment of diabetic mice with GSPB2 significantly decreased whole body weight and serum lipid levels, as well as hepatic lipid droplet accumulation and hepatic TG and FFA levels. GSPB2 restored liver AMPK and ACC phosphorylation levels, increased CPT1 protein levels and minimized the subsequent damage from lipid peroxidation. Furthermore, GSPB2 treatment significantly 
A

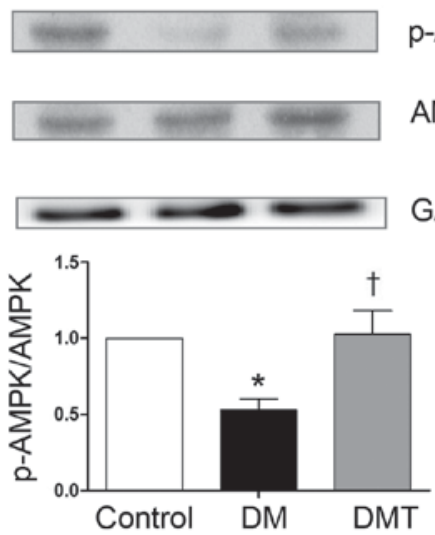

B

\section{P-AMPK \\ AMPK}

GAPDH

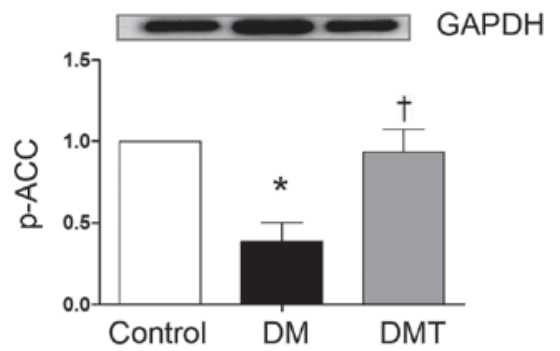

Figure 3. GSPB2 restores liver AMPK and ACC phosphorylation levels in diabetic mice. Representative western blot analysis of (A) p-AMPK and total AMPK and (B) p-ACC subunits ACC $\alpha$ at $265 \mathrm{kDa}$ and $\mathrm{ACC} \beta$ at $280 \mathrm{kDa}$. Data are presented as the mean + standard error (n=6 per group). " $\mathrm{P}<0.05 \mathrm{vs}$. control and ${ }^{\dagger} \mathrm{P}<0.05$ vs. DM. GSPB2, grape seed procyanidin B2; AMPK, AMP-activated protein kinase; ACC, acetyl-CoA carboxylase; p-; phosphorylated; DM, diabetic mice treated with vehicle alone; DMT, DM treated with GSPB2.

A

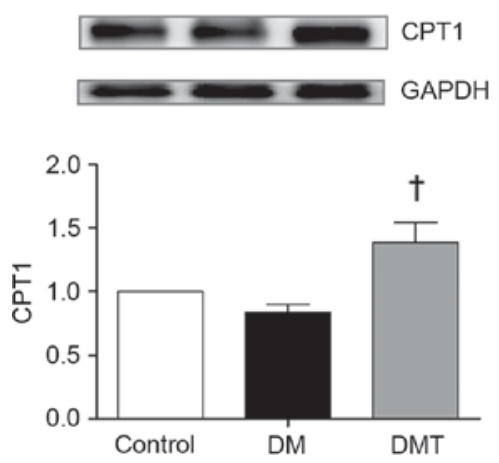

B
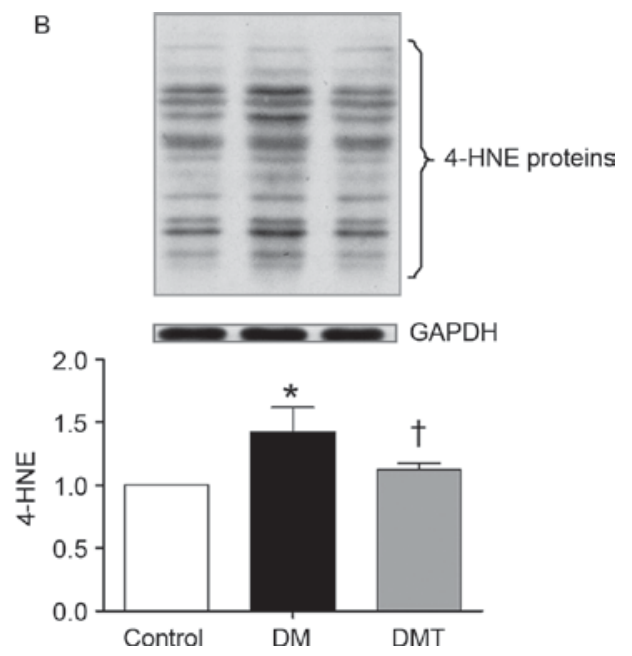

Figure 4. GSPB2 increases CPT1 protein levels and ameliorates lipid peroxidation damage in diabetic mice. Representative western blot analysis of expression of (A) CPT1 and (B) 4-HNE proteins. 4-HNE protein levels were used as a marker of cellular lipid peroxidation damage. Data are presented as the mean + standard error ( $\mathrm{n}=6$ per group). ${ }^{*} \mathrm{P}<0.05$ vs. control and ${ }^{\dagger} \mathrm{P}<0.05$ vs. DM. GSPB2, grape seed procyanidin B2; CPT1, carnitine palmitoyl transferase 1; 4-HNE, 4-hydroxynonenal; DM, diabetic mice treated with vehicle alone; DMT, DM treated with GSPB2.

ameliorated hepatic mitochondrial dysfunction and restored mitochondrial CI- and CII-dependent respiratory capacity. The results of the present study indicate that long-term oral treatment with GSPB2 may benefit hepatic lipid metabolism disorders, potentially by increasing hepatic FFA $\beta$-oxidation in mitochondria and decreasing hepatic lipid synthesis via AMPK-ACC-mediated lipid metabolism.

Although GSPB2 did not significantly reduce FBG, it normalized serum and hepatic elevated lipid levels and reduced body weight gain in $\mathrm{db} / \mathrm{db}$ mice. These results indicate that GSPB2 treatment predominantly affects lipid, but not glucose, metabolism, suggesting that its main benefit in diabetes may occur through a potential protective effect against atherosclerosis and cardiovascular diseases $(24,25)$. The liver is the major site for the storage and release of carbohydrates, and the synthesis of FAs. In this diabetic model, liver lipid accumulation and lipid metabolic disorders were alleviated by GSPB2 treatment, therefore, GSPB2 may be an effective

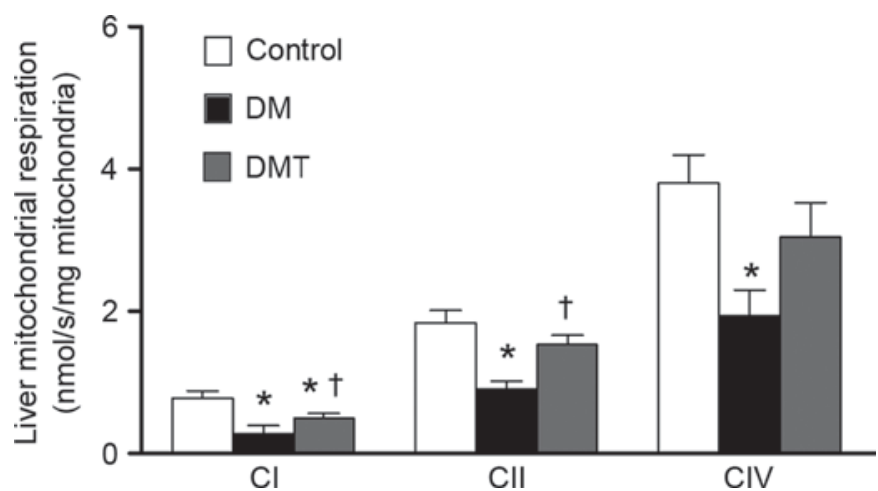

Figure 5. GSPB2 restores the damaged liver mitochondrial respiratory capacity in diabetic mice. Data are presented as the mean \pm standard error $(n=4)$. ${ }^{*} \mathrm{P}<0.05$ vs. control and ${ }^{\dagger} \mathrm{P}<0.05$ vs. DM. GSPB2, grape seed procyanidin B2; CI, electron transport complex I; CII, electron transport complex II; CIV, electron transport complex IV; DM, diabetic mice treated with vehicle alone; DMT, DM treated with GSPB2. 
agent to improve lipid metabolism, decelerate the progression of diabetes complications and improve prognosis in diabetes.

In order to uncover the underlying mechanisms of GSPB2 on liver lipid metabolism, the current study investigated the potential involvement of AMPK. AMPK has a key role in regulating lipid metabolism through multiple signaling pathways, including directly catalyzing its downstream substrates and the transcription of multiple genes (26). AMPK is activated $>200$-fold by phosphorylation at Thr172 (7). Similar to the results of a previous study (27), the present study demonstrated that phosphorylated AMPK levels were lower in DM mice compared with controls. Several other studies have demonstrated that GSPE treatment ameliorated mitochondrial dysfunction and inhibited oxidative stress or apoptosis via AMPK-dependent signaling $(17,18)$. Similarly, in the present study, GSPB2 treatment significantly restored the hepatic phosphorylation of AMPK. The activation of AMPK may lead to the stimulation of FA oxidation and inhibition of lipogenesis (9), reduced hepatic lipid accumulation and, in turn, attenuated hyperlipidemia and atherosclerosis in DM (12).

In the present study, from the H\&E-stained paraffin sections and liver homogenate lipid measurements, lipid synthesis and accumulation in the liver were demonstrated to be reduced in diabetes, possibly by inactivation of ACC by AMPK. The phosphorylation of ACC was lower in DM mice compared with controls, however, ACC phosphorylation was restored by GSPB 2 treatment. ACC is a key downstream target of AMPK and catalyzes the production of malonyl-CoA from acetyl-CoA, which is a key intermediate of FA synthesis and oxidation (10). Increased AMPK activity phosphorylates and inactivates ACC $\alpha$ (at Ser79) and ACC $\beta$ (at Ser221) in the liver, which results in reduced malonyl-CoA production (28) and may lead to reduced hepatic FA synthesis (9).

CPT1 is the rate-limiting enzyme that transfers the long-chain fatty acyl CoA to mitochondria for $\beta$-oxidation (11). It has been implicated as contributing to elevated FFA levels, fat accumulation and decreased ability to oxidize FAs in diabetes. The increased levels of malonyl-CoA caused by ACC activation inhibit CPT1, leading to a subsequent decrease in the transport of long-chain FAs into mitochondria and decreased FA oxidation $(29,30)$. The shunting of long-chain FAs away from mitochondria leads to an increase in FFA levels and the accumulation of fat $(29,30)$. In the current study, GSPB2 significantly restored the decreased CPT1 level in DM and increased FA movement as fuel into mitochondria for $\beta$-oxidation, which would lead to increased oxygen consumption to generate ATP. The mitochondrial CI- and CCII-dependent respiratory capacity was significantly reduced in DM mice compared with controls, however, this was significantly restored by GSPB2 treatment, which may be due to increased fuel supply for mitochondrial ATP production or ameliorated lipid peroxidation.

GSPB2 significantly decreased hepatic FFA levels and minimized subsequent lipid peroxidation damage, as measured by 4-HNE proteins levels. 4-HNE is a measure of lipid peroxidation from reactive oxygen species (ROS). Elevated FFA levels increase ROS production in diabetes and obesity (31), which exacerbates mitochondrial dysfunction. ROS directly damages mitochondrial proteins and promotes the formation of the mitochondrial permeability transition pore, increases the release of cytochrome c and enhances apoptosis (32). Injured mitochondria cannot effectively reduce oxygen or transfer electrons, which results in high levels of ROS that further damage tissues, trigger apoptosis and lead to a vicious cycle of ROS production and mitochondrial dysfunction (33).

Grape seed extract is generally well tolerated when taken orally. It has been used safely for up to 8 weeks in clinical trials (34). Administration of the grape seed extract to male and female Sprague Dawley rats in the feed at levels of 0.5 , 1.0 , or $2.0 \%$ for 90 days did not induce any significant toxicological effects (35). In the present study and using GSPB2 at a dose of $30 \mathrm{mg} / \mathrm{kg}$ body weight per day orally for 10 weeks, no obvious toxicity to the mice was observed. However, certain side effects of grape seed extract have been reported, including a dry, itchy scalp, dizziness, headache, high blood pressure, hives, indigestion and nausea (34). Consumption of grape seed extract polyphenols may inhibit non-heme iron absorption and may lead to iron depletion in populations with marginal iron stores, and also may interact with certain pharmaceutical agents and enhance their biologic effects (36). A higher dose of grape seed extract may lead to increased toxicity to animals, and this requires further investigation. In the current study, the effects of GSPB2 on hepatic lipid metabolism disorders in diabetic mice were investigated. GSPB2 decreased hepatic lipid droplet accumulation and hepatic lipid levels. These protective effects appear to be mediated by increased hepatic FFA $\beta$-oxidation in mitochondria and decreased hepatic lipid synthesis by stimulating an AMPK-ACC lipid metabolic pathway. In order to fully investigate the potential molecular mechanism, experiments with either antagonists of AMPK or an AMPK knock-out mouse strain would be required in future studies. Overall, GSPB2 may represent a novel therapeutic agent to improve whole-body lipid metabolism, decelerate the progression of diabetes complications and improve the prognosis of patients with diabetes.

\section{Acknowledgements}

This work was supported by grants from the National Natural Science Foundation of China (grant no. 81501786), the Major Projects of National Science and Technology of China (grant no. 2012ZX09303016-003) and the Horizontal Project of Shandong University (grant no. 11671614). The sponsor of the funding had no active role in the design, methods, data collections, analysis, or preparation of this manuscript or in the decision to submit the manuscript for publication. We thank Ms. Laura Smales (BioMedEditing, Toronto, ON, Canada) for language editing of this manuscript.

\section{References}

1. American Diabetes Association: Economic costs of diabetes in the U.S. in 2012. Diabetes Care 36: 1033-1046, 2013.

2. Ginter E and Simko V: Type 2 diabetes mellitus, pandemic in 21st century. Adv Exp Med Biol 771: 42-50, 2012.

3. Goldberg IJ: Clinical review 124: Diabetic dyslipidemia: Causes and consequences. J Clin Endocrinol Metab 86: 965-971, 2001.

4. Yan L, Xu MT, Yuan L, Chen B, Xu ZR, Guo QH, Li Q, Duan Y, Huang Fu J, Wang YJ, et al: Prevalence of dyslipidemia and its control in type 2 diabetes: A multicenter study in endocrinology clinics of China. J Clin Lipidol 10: 150-160, 2016.

5. Bhatt HB and Smith RJ: Fatty liver disease in diabetes mellitus. Hepatobiliary Surg Nutr 4: 101-108, 2015. 
6. Stefan N and Häring HU: The metabolically benign and malignant fatty liver. Diabetes 60: 2011-2017, 2011

7. Hardie DG: AMP-activated protein kinase: An energy sensor that regulates all aspects of cell function. Genes Dev 25: 1895-1908, 2011.

8. Hou X, Xu S, Maitland-Toolan KA, Sato K, Jiang B, Ido Y, Lan F, Walsh K, Wierzbicki M, Verbeuren TJ, et al: SIRT1 regulates hepatocyte lipid metabolism through activating AMP-activated protein kinase. J Biol Chem 283: 20015-20026, 2008

9. Viollet B, Foretz M, Guigas B, Horman S, Dentin R, Bertrand L, Hue L and Andreelli F: Activation of AMP-activated protein kinase in the liver: A new strategy for the management of metabolic hepatic disorders. J Physiol 574: 41-53, 2006.

10. Wong AK, Howie J, Petrie JR and Lang CC: AMP-activated protein kinase pathway: A potential therapeutic target in cardiometabolic disease. Clin Sci (Lond) 116: 607-620, 2009.

11. Ruderman NB, Saha AK and Kraegen EW: Minireview: Malonyl CoA, AMP-activated protein kinase and adiposity. Endocrinology 144: 5166-5171, 2003.

12. Zang M, Xu S, Maitland-Toolan KA, Zuccollo A, Hou X, Jiang B, Wierzbicki M, Verbeuren TJ and Cohen RA: Polyphenols stimulate AMP-activated protein kinase, lower lipids and inhibit accelerated atherosclerosis in diabetic LDL receptor-deficient mice. Diabetes 55: 2180-2191, 2006

13. Terra X, Montagut G, Bustos M, Llopiz N, Ardèvol A, Bladé C Fernández-Larrea J, Pujadas G, Salvadó J, Arola L and Blay M: Grape-seed procyanidins prevent low-grade inflammation by modulating cytokine expression in rats fed a high-fat diet. J Nutr Biochem 20: 210-218, 2009.

14. Sharma SD and Katiyar SK: Dietary grape seed proanthocyanidins inhibit UVB-induced cyclooxygenase-2 expression and other inflammatory mediators in UVB-exposed skin and skin tumors of SKH-1 hairless mice. Pharm Res 27: 1092-1102, 2010.

15. Pajuelo D, Díaz S, Quesada H, Fernández-Iglesias A, Mulero M, Arola-Arnal A, Salvadó MJ, Bladé C and Arola L: Acute administration of grape seed proanthocyanidin extract modulates energetic metabolism in skeletal muscle and BAT mitochondria J Agric Food Chem 59: 4279-4287, 2011.

16. Mansouri E, Khorsandi L and Abedi HA: Antioxidant effects of proanthocyanidin from grape seed on hepatic tissue injury in diabetic rats. Iran J Basic Med Sci 17: 460-464, 2014.

17. Bao L, Cai X, Zhang Z and Li Y: Grape seed procyanidin B2 ameliorates mitochondrial dysfunction and inhibits apoptosis via the AMP-activated protein kinase-silent mating type information regulation 2 homologue 1-PPAR $\gamma$ co-activator-1 $\alpha$ axis in rat mesangial cells under high-dose glucosamine. Br J Nutr 113: 35-44, 2015

18. Bao L, Cai X, Dai X, Ding Y, Jiang Y, Li Y, Zhang Z and Li Y: Grape seed proanthocyanidin extracts ameliorate podocyte injury by activating peroxisome proliferator-activated receptor- $\gamma$ coactivator $1 \alpha$ in low-dose streptozotocin-and high-carbohydrate/high-fat diet-induced diabetic rats. Food Funct 5: $1872-1880,2014$

19. Han S, Jiao J, Zhang W, Xu J, Wan Z, Zhang W, Gao X and Qin L: Dietary fiber prevents obesity-related liver lipotoxicity by modulating sterol-regulatory element binding protein pathway in C57BL/6J mice fed a high-fat/cholesterol diet. Sci Rep 5: 15256, 2015.

20. Palmeira CM and Moreno AJ : Mitochondrial bioenergetics: Methods and protocols. In: High-resolution respirometry. OXPHOS protocols for human cells and permeabilized fibers from small biopsies of human muscle. Pesta D and Gnaiger E (eds). Humana Press, New York, NY, pp25-58, 2012.

21. Kuznetsov AV, Veksler V, Gellerich FN, Saks V, Margreiter R and Kunz WS: Analysis of mitochondrial function in situ in permeabilized muscle fibers, tissues and cells. Nat Protoc 3 965-976, 2008.
22. Karamercan MA, Weiss SL, Villarroel JP, Guan Y, Werlin E, Figueredo R, Becker LB and Sims C: Can peripheral blood mononuclear cells be used as a proxy for mitochondrial dysfunction in vital organs during hemorrhagic shock and resuscitation? Shock 40: 476-484, 2013.

23. Zhang Z, Li BY, Li XL, Cheng M, Yu F, Lu WD, Cai Q, Wang JF, Zhou RH, Gao HQ and Shen L: Proteomic analysis of kidney and protective effects of grape seed procyanidin $\mathrm{B} 2 \mathrm{in} \mathrm{db} / \mathrm{db}$ mice indicate MFG-E8 as a key molecule in the development of diabetic nephropathy. Biochim Biophys Acta 1832: 805-816, 2013.

24. Yu F, Li BY, Li XL, Cai Q, Zhang Z, Cheng M, Yin M, Wang JF, Zhang JH, Lu WD, et al: Proteomic analysis of aorta and protective effects of grape seed procyanidin B2 in $\mathrm{db} / \mathrm{db}$ mice reveal a critical role of milk fat globule epidermal growth factor- 8 in diabetic arterial damage. PLoS One 7: e52541, 2012

25. Luan SS, Yu F, Li BY, Qin RJ, Li XL, Cai Q, Yin WB, Cheng M and Gao HQ: Quantitative proteomics study of protective effects of grape seed procyanidin B2 on diabetic cardiomyopathy in db/db mice. Biosci Biotechnol Biochem 78: 1577-1583, 2014.

26. Hardie DG: The AMP-activated protein kinase pathway-new players upstream and downstream. J Cell Sci 117: 5479-5487, 2004.

27. Viollet B, Lantier L, Devin-Leclerc J, Hebrard S, Amouyal C, Mounier R, Foretz M and Andreelli F: Targeting the AMPK pathway for the treatment of Type 2 diabetes. Front Biosci (Landmark Ed) 14: 3380-3400, 2009.

28. Kim MK, Kim SH, Yu HS, Park HG, Kang UG, Ahn YM and Kim YS: The effect of clozapine on the AMPK-ACC-CPT1 pathway in the rat frontal cortex. Int J Neuropsychopharmacol 15: 907-917, 2012.

29. Rasmussen BB, Holmbäck UC, Volpi E, Morio-Liondore B Paddon-Jones D and Wolfe RR: Malonyl coenzyme A and the regulation of functional carnitine palmitoyltransferase-1 activity and fat oxidation in human skeletal muscle. J Clin Invest 110: 1687-1693, 2002.

30. McGarry JD, Mills SE, Long CS and Foster DW: Observations on the affinity for carnitine and malonyl-CoA sensitivity, of carnitine palmitoyltransferase I in animal and human tissues. Demonstration of the presence of malonyl-CoA in non-hepatic tissues of the rat. Biochem J 214: 21-28, 1983.

31. Inoguchi T, Li P, Umeda F, Yu HY, Kakimoto M, Imamura M, Aoki T, Etoh T, Hashimoto T, Naruse M, et al: High glucose level and free fatty acid stimulate reactive oxygen species production through protein kinase $\mathrm{C}$-dependent activation of NAD $(\mathrm{P}) \mathrm{H}$ oxidase in cultured vascular cells. Diabetes 49 : 1939-1945, 2000.

32. Skripchenko A, Myrup A, Thompson-Montgomery D, Awatefe H, Moroff G and Wagner SJ: Periods without agitation diminish platelet mitochondrial function during storage. Transfusion 50: 390-399, 2010

33. Cuzzocrea S, Riley DP, Caputi AP and Salvemini D: Antioxidant therapy: A new pharmacological approach in shock, inflammation, and ischemia/reperfusion injury. Pharmacol Rev 53: 135-159, 2001.

34. Sovak M: Grape Extract, Resveratrol and Its Analogs: A Review. J Med Food 4: 93-105, 2001.

35. Wren AF, Cleary M, Frantz C, Melton S and Norris L: 90-day oral toxicity study of a grape seed extract (IH636) in rats. J Agric Food Chem 50: 2180-2192, 2002.

36. Mennen LI, Walker R, Bennetau-Pelissero C and Scalbert A: Risks and safety of polyphenol consumption. Am J Clin Nutr 81 (1 Suppl): S326-S329, 2005. 